the capsule with a pair of catch forceps and drawing it into the wound at the same time as pressure was made on the abdominal surface by an assistant. The fibrous covering of the pelvis was then opened with a bistoury, and a large mulberry calculus extracted by aid of a scoop. Nineteen smaller separate calculi were subsequently removed which lay in the pelvis and calyces. The secreting structure of the kidney did not appear to have been very extensively damaged or diminished. There was not much hæmorrhage, and, the wound having been washed out with warm boracic lotion, a drainage-tube was inserted into the pelvis and the wound united by deep and superficial sutures. The parts were dressed with sal alembroth ganze and covered with wood wool. The patient was in considerable pain after recovering from the ether, and reqired injections of morphia. The discharges permeated the dressings, which were packed but not changed. At a quarter to eight a catheter was passed, and sixteen ounces of blood-stained urine were withdrawn. No vomiting. Temperature $98.4^{\circ}$; pulse 88 . The catheter was required next day, but on July 6 th the urine was passed naturally, and contained deep red sediment consisting of blood and pus-cells. The wound was perfectly healthy. For the next few days less and less urine came through the wound, and on the 10th the tube and stitches were removed, the wound looking perfectly healthy. On the 11 th he had a slight rigor, the temperature rising to $101^{\circ}$, and on the 12 th a more severe one, when the thermometer marked $104^{\circ}$, but there was nothing in the appearance of the wound to cause anxiety. These symptoms did not recur, and the patient went on perfectly well, and was discharged from the hospital with the wound firmly healed on July $28 \mathrm{th}$. The larger of the calculi weighed 141 grains, and was irregularly conoidal in shape, the blunted apex having projected into the commencement of the ureter, whilst the base filled a large portion of the pelvis of the kidney. The colour was a deep madder brown on the surface, and a few crystalline deposits of phosphate of lime adbered to one or two spots. The surface was irregularly mammillated, and there were no very sharp projections. The total weight of the calculi amounted to 156 grains, the remaining stones being all separate and unbroken, and resembling in shape and appearance a number of small "sea urchins." The composition as ascertained by analysis was pure oxalate of lime.

\section{SOUTH DEVON AND EAST CORNWALL HOSPITAL, PLYMOUTH.}

A SHORT SERIES OF RENAL CASES; REMARKS.

DURING the last two or three years our knowledge of the operative treatment of many diseases of the kidney has considerably extended, and much advance has been made in this department of surgery. Two of this series of cases are of special interest. In the first, that of hydronephrosis in a woman (and hydronephrosis is more common in females), the usual treatmient having failed, excision of the kidney was successfully performed and the patient cured. In the second, where it is probable that a partial rupture of the kidney had resulted from the injury described, simple aspiration without drainage sufficed for a cure. We have published this year other interesting renal cases from this hospital, ${ }^{1}$ and for them and for the notes of these cases we are indebted to Mr. W. Gifford Nash, house surgeon.

CASE 1. Hydronephrosis; nephrotomy ; renal fistula nephrectomy; recovery. (Under the care of Mr. WHIPPLE.)A. M — aged forty-two, a married woman, was admitted on Aug. 5th, 1890. She stated that two years previously, whilst lifting a piano, she felt something give way in the left loin. She vomited at the time, and soon afterwards noticed a lump in the left half of the abdomen, which gradually increased and gave very little pain. About once a month she had an attack of vomiting, followed by decrease in the size of the lump, and an increase in the amount of urine passed.

On admission there was found in the left loin an elastic, rounded, fluctuating swelling, reaching up under the costal margin, and downwards a little below the umbilicus. There was bulging in the ilio.costal space. An aspirating needle was introduced, and a fluid of neutral reaction and free from albumen drawn off.

1 THE LaNCET, vol. i. 1891, pp. 1070-1123.
On Aug. 16th lumbar nephrotomy was performed, and the pelvis of the kidney explored. It was enormonsly dilated, holding three pints of urine, but no cause was found. The kidney was drained, but showed no disposition to heal. Urine containing pus and albumen drained from the loin, but the urine from the bladder was normal.

On Nov. 7th a loin urinal was fitted, and next day the patient left the hospital. She was kept under observation. The urinal worked well, but the patient gradually lost flesh probably due to the constant drain of albumen in the urine from the left loin. The daily average of urine from the lef loin was about fifteen ounces, and from the bladder thirty. five ounces.

On May 19th, 1831, the patient was readmitted for nephrectomy. The daily average of urine for ten days previous to operation was from the loin thirteen ounces, and from the bladder thirty-two ounces. On June 8th the left kidney was removed through the loin. The pelvis and calyces of the kidney were considerably dilated, and th cortical substance pale, thin, and fibrous. The wal of the pelvis was thickened and the mucous membrane roughened The ureter was of natural size up to its junction with the pelvis, where it appeared to be narrowed, as if the result of a bend or kink. The patient left the hospital quite well on July 6 th. The daily average of urine for three weeks after the operation was thirty. one ounces.

Remarls. - What the exact nature of the constriction was could not be ascertained, as the ureter unfortunately broke away from the pelvis at the operation. It seemed probable tbat it was either a twist or kink of the ureter of a movable kidney, or else a partial rupture of the coats of the ureter at its junction with the kidney pelvis. At ang rate, the obstruction was at the junction of the pelvis and ureter, and its probable cause was the strain due to lifting the piano.

CASE 2. Traumatic renal cyst; aspiration; cure. (Undey the care of Mr. SwAIN.)-E. B-, aged six, was admitted on October 21 st, 1890 . It was stated that five months previously the boy fell and struck his right side against a bucket. A few weeks later his mother found a lump on the right side of the abdomen. This increased rapidly.

On admission, he was a well-nourished, rickety child. In the right half of his abdomen was a firm swelling extending into the loin and continuous with the kidney. Intestine lay in front of it. It extended up under the ribs, but between it and the liver was a sulcus. Fluctuation could not be obtained. The urine was acid and free from albumen An exploratory operation was proposed, but the parents refused to consent to one. He left on Oct. 28th, but remained under observation. As he got no worse and the lump remained unaltered, on April 14th, 1891, he was re admitted. On the 16 th the tumour was aspirated througb the right loin, and thirty-one ounces of pale, almost colour less fluid, with a specific gravity of 1009 , drawn off This fluid contained very little urea, and under the micro scope were seen a few blood.cells. When the fluid wa drawn off the kidney could be felt, not at all enlarged There has been no sign of reaccumulation of thaid.

Remarks. - This appears to have been a case of peri-resal urinary cyst, probably due to rupture of the pelvis of the kidney. At first it was thought that it might be a malignant growth, but the stationary condition of the growth and the absence of constitutional symptoms negatived this.

CASE 3. Pernicious ancemia; single lidney. (Under the care of Dr. PRANCE, -J. McD__, aged sixty, a sailor, was admitted on Feb. 11th, 1891, and died on March 5th. $\mathrm{He}$ had all the typical symptoms of pernicions anæmia. At the post mortem examination it was found that the left kidney was very much enlarged, in the usual position, and with normal bloodvessels. There was not the siightest trace of the right kidney or ureter. A very small artery, appa rently representing the right renal, was distributed on the posterior surface of the ascending colon.

\section{LEEDS GENERAL INFIRMARY.}

A CASE OF SPONTANEOUS RUPTURE OF THE BLADDER.

(Under the care of Mr. W. H. BrowN.)

THIs case, a very unusual one of extra-peritoneal rupture of the bladder, presented difficulties in diagnosis which could not be overcome in the absence of history. Even with the light thrown on the case by the 
post-mortem examination, it is difficult to account for the giving way of the bladder. Stricture of the urethra, with alteration in the normal structure of the bladder, is recognised as a predisposing cause for rupture, and a stricture was found; but the stricture is usually one producing marked obstruction to the flow of urine, and the changes in the bladder wall are easily seen. These changes include: (l) the formation of diverticula of the mucous membrane, with hypertrophy of the muscular coat; (2) ulceration, sloughing, ur gangrene of the mucous membrane secondary to a cystitis; (3) thinning and weakening of the coats of the bladder. ${ }^{1}$ The only evidence of rupture of the bladder was that produced by the cellulitis resulting from the escape of urine into the subperitoneal tissues. There was no account forthconing of a likely injury; no inability to micturate; no trace of blood in the urine; the catheter when passed drew off clear urine, showing the bladder was not unduly contracted. ${ }^{2}$

S. S_- a mechanic, aged thirty-nine, was admitted to the infirmary on June $29 \mathrm{th}, 189 \mathrm{l}$, in a dying condition. The abdomen was much distended and tympanitic, with the exception of the left iliac region, which was dull; the skin over this portion of the abdomen was slightly oedematous and discoloured. The man was too ill to give any account of himself, and as the distention appeared to be the chief cause of his distress, a small incision was made in the middle line, giving exit to a large quantity of coffeecoloured fluid, which had a distinctly urine-like odour. Before anything further could be done the patient died, apparently from heart failure. His history, afterwards obtained from the friends, was as follows. The deceased had always been a healthy man, leading an active life, playing cricket up to a week before admission. On June 22 nd he began to have a little pain in the body, but did not consult his doctor till a day or two later, when he began to vomit. He was then seen by his usual medical attendant, who found the abdomen distended and signs of intestinal obstruction. These continued in spite of treatment. No history of accident of any kind could be elicited. A catheter was passed before he was sent to the inficmary, and clear urine withdrawn.

On post-mortem examination, the incision made before death opened into a large cavity between the peritoneum and the abdominal wall. This cavity extended upwards towards the lefo kidney, and contained a quantity of flaid similar to that which was let out before death. At the extreme limit of the cavity there were distinct signs of suppuration. At the fund us of the bladder there was a small opening, half an inch in diameter, leading in to the cavity, formed by the stripping of the peritoneum. The edges of the bladder opening were rounded off and smooth; there was no sign of recent bleeding. The peritoneal cavity contained abant two piats of serous fluid; the intestines were greatly distended; there was no mechanical obstruction. There was a stricture of the urethra of old standing capable of admitting a No. 6 catheter. The bladder was a little thickened, but otherwise natural. The body was, generally speaking, well nourished, and the viscera healthy.

Remarks by Mr. Brown.-In THE LANCET, vol. ii 1888, p. 208, I published the notes of a case of rupture of the bladder ocsurring in a healthy man, where the history threw no light on the condition. In the present case the rupture was extra-peritoneal, and the symptoms remained in abeyance for some days after the probable time of giving way of the bladder. It seems likely that at the actual time of rupture some blood would have bren passed by the nrethra; of this, however, no history could be obtained. In both of these cases the position of the rupture corresponded with the spot upon which the point of a catheter, introduced into the bladder, would impinge.

1 Rivington: Heath's Dictionary of Surgery. Jacobson: The Operations of Surgery, 2nd edition, p. 918

Rabies IN NoRTHAMPTONSHIRH.-At a meeting of the Executive Committee of the County Council of Northamptonshire, Earl Spencer in the chair, it was decided to issue muzzling orders for nineteen parishes. This course was rendered necessary owing to the ravages of a mad dog, which had before it was killed bitten twelve other animals, including two valuable fox.hounds belonging to the Pytchley Hunt.

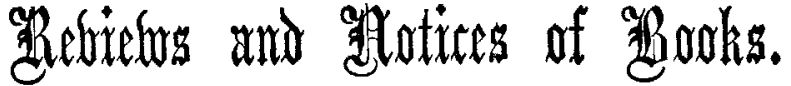

Diphtheria; its Nature and Treatment By RoBERT

William PARKer. Third Edition, largely rewritten. London : H. K. Lewis. 1891.

THE admitted value of this excellent monograph has been enhanced by the introduction of much new matter into this edition. Mr. Parker has prefixed to his description of the operation of tracheotomy and all that surrounds it a brief account of present knowledge concerning the nature of diphtheria and its manifestations. He declares himself con vinced that the disease has a germ origin, that it is essentially local at first, and then becomes generally infective-a view borne out strongly by Oertel's elaborate histological studies, to which Mr. Parker adverts. The adoption of this idea of its nature harmonises with another view held by Mr. Parker, that, namely, of a local predisposition. He says that he has of ten met with sporadic cases in families where the oppor. tunities for thorough sanitary precautions have been of the smallest, and that he is quite at variance with the view that there is any special family proclivity to the disease. Nevertheless, since he lays such great stress upon local conditions of throat-pharyngitis, tonsillitis, post-nasal growths, \&c.as predisponent to infection, or rather as affording a certain receptivity to the contagion, it is not extravagant to assume, for members of the same family, a similarity in such local conditions. That diphtheria is primarily local and secondarily systemic is, we believe, almost beyond question. It is a conception of its nature that affords most encouragement for early and thorough local treatment; and it accords with the clinical development of the disease as well as with modern investigations upon the diphtherial virus. Mr. Parker's description of the symptoms of diphtheria and its complications is exact; but it might with advantage have been more fully elaborated. We note that he attributes (p. 47) cardiac failure or syncope to paralysis of the vagus nerve; and, without gainsaying the accuracy of this view, we think it should be held with some reserve; for we are not cognisant of any definite facts (anatomical) in support of this contention. On the other hand, that the cardiac muscle may be profoundly affected by the diph theritic poison is indubitable. No doubt there is truth in both interpretations of sudden death in diphtheria, an event which we agree with Mr. Parker to be "very common."

The reader will turn with most interest to the chapters devoted to tracheotomy and its after-treatment, in the hope of finding there the fruits of the author's recent experience. And he will not be disappointed. It seems to us that $\mathrm{Mr}$. Parker has materially modified several of his views and procedures, all in the way of improvement. Most important is his adoption of what may be called the "rapid opera tion "-that in which the trachea is opened by two incisions, the first through the skin and fascia, and the second into the trachea, guided by the intermuscular "white line" which occupies the centre of the neck. In Mr. Parker's hands such an operation has doubtless advantages, but for the majority the risks of hæemorrhage and of emphysema may be more surely obviated by more deliberate dissection. The angular tube invented by the author, and shown by him to be in every respect better adapted to the form and postion of the trachea, is a distinct advance upon all other forms, and should be generally adopted. Every detail of the operation here described shows the fertility in resource of the writer, who, amongst other forms of apparatus, has invented a trachea aspirator for the removal of material obstructing the trachea or bronchus. He condemns as useless and hazardous the direct application of the operator's 